\title{
An iterative method for variational inequality problems
}

Wei-Bo Guan*

\section{"Correspondence:}

guanweibomath@163.com

Department of Mathematics, Harbin

Institute of Technology, Harbin,

150001, P.R. China

School of Mathematical Sciences,

Harbin Normal University, Harbin

150025, P.R. China

\section{Abstract}

In this paper, we present some properties of generalized proximity operators and propose an iterative method of approximating solutions for a class of generalized variational inequalities and show its convergence in uniformly convex and smooth Banach spaces.

MSC: 47J20; 46B20; 46N10; 47N10; 49J40

Keywords: Banach space; proximity operator; variational inequality; iterative method

\section{Introduction}

Let $f$ be a lower semi-continuous proper convex function from a Hilbert space $H$ to $(-\infty,+\infty]$. The Moreau envelope of the function $f$ is defined as

$$
e^{f}(x)=\inf _{y \in H}\left\{f(y)+\frac{1}{2}\|x-y\|^{2}\right\} .
$$

It is well known that $e^{f}(x)$ is a continuous convex function, and for every $x \in H$, the infimum in (1.1) is achieved at a unique point $\operatorname{prox}_{f}(x)$. The operator $\operatorname{prox}_{f}$ from $H$ to $H$, i.e.,

$$
\operatorname{prox}_{f} x=\underset{y \in H}{\arg \min }\left\{f(y)+\frac{1}{2}\|x-y\|^{2}\right\}
$$

thus defined, is called the proximity operator of $f$. When $f=\iota_{K}$ is the indicator function of a closed convex set $K$ in $H$, then $\operatorname{prox}_{f}(x)=P_{K}(x)$ becomes the metric projection operator on $K$.

In 1994, Alber extended the metric projection operator to uniformly convex and uniformly smooth Banach spaces. Let $K$ be a closed convex subset of a uniformly convex and uniformly smooth Banach space $X$, Alber [1] introduced the generalized projections $\pi_{K}: X^{*} \rightarrow K$ and $\Pi_{K}: X \rightarrow K$

$$
\pi_{K}\left(x^{*}\right)=\underset{x \in K}{\arg \min }\left\{\left\|x^{*}\right\|^{2}-2\left\langle x^{*}, x\right\rangle+\|x\|^{2}\right\}
$$

and

$$
\Pi_{K}(x)=\underset{y \in K}{\arg \min }\left\{\|J x\|^{2}-2\langle J x, y\rangle+\|y\|^{2}\right\},
$$

(อ2013 Guan; licensee Springer. This is an Open Access article distributed under the terms of the Creative Commons Attribution License (http://creativecommons.org/licenses/by/2.0), which permits unrestricted use, distribution, and reproduction in any medium, provided the original work is properly cited. 
where $J$ is the duality mapping from $X$ to $X^{*}$, and studied their properties in detail. In [2], Alber presented some applications of the generalized projections to approximately solving variational inequalities in Banach spaces. Recently, Li [3] extended the generalized projection operator $\pi_{K}$ from uniformly convex and uniformly smooth Banach spaces to reflexive Banach spaces and studied some properties of the generalized projection operator with applications to solving the variational inequality in Banach spaces. By employing the generalized projection operators, Zeng and Yao [4] established some existence results for the variational inequality problem in uniformly convex and uniformly smooth Banach spaces and convergence results for the variational inequality. In [5], Wu and Huang further introduced and studied a class of generalized $f$-projection operators in Banach spaces. As applications, they proposed an iterative method of approximating solutions for the variational inequality problem: find $\bar{x} \in K$ such that

$$
\langle A \bar{x}, y-\bar{x}\rangle+f(y)-f(\bar{x}) \geq 0, \quad \forall y \in K
$$

where $K$ is a nonempty closed convex subset of $X, A: K \rightarrow X^{*}$ is a mapping and $f: X \rightarrow$ $(-\infty,+\infty]$ is a proper convex, lower semicontinuous and positively homogeneous function, via

$$
x_{n+1}=\pi_{K}^{f}\left(J x_{n}-\alpha_{n} J\left(x_{n}-\pi_{K}^{f}\left(J x_{n}-\rho A x_{n}\right)\right)\right),
$$

where

$$
\pi_{K}^{f}\left(x^{*}\right)=\underset{x \in K}{\arg \min }\left\{2 \rho f(x)+\left\|x^{*}\right\|^{2}-2\left\langle x^{*}, x\right\rangle+\|x\|^{2}\right\},
$$

and the parameter sequence $\left\{\alpha_{n}\right\}$ satisfies

$$
0 \leq \alpha_{n} \leq 1, \quad \sum_{n=0}^{+\infty} \alpha_{n}\left(1-\alpha_{n}\right)=+\infty, \quad \rho>0,
$$

and they proved that $\left\{x_{n}\right\}$ has a subsequence converging to a solution of (1.3) when $K$ is a nonempty compact convex subset of a uniformly convex and uniformly smooth Banach space.

Motivated and inspired by the above works, we continue to study some properties of generalized proximity operators and propose an iterative method of approximating solutions for the following generalized variational inequality problem: find $\bar{x} \in \operatorname{dom} f$ such that

$$
\langle A \bar{x}, y-\bar{x}\rangle+f(y)-f(\bar{x}) \geq 0, \quad \forall y \in \operatorname{dom} f,
$$

where $f: X \rightarrow(-\infty,+\infty]$ is a proper convex and lower semicontinuous function, $A: X \rightarrow$ $X^{*}$ is a norm-to-weak continuous operator. Our iterative method is different from that given in [5]. We also prove a convergence result for this iterative method in smooth and uniformly convex Banach spaces. Let $K$ be a nonempty closed convex set of $X$. If we replace $f$ by $f+I_{K}$ in (1.5), where $I_{K}$ is the indicator function of $K$, then (1.5) reduces to (1.3). 


\section{Preliminaries}

Let $X$ be a reflexive, smooth and strictly convex Banach space with the dual space $X^{*}$. We denote by $x_{n} \rightarrow x$ and $x_{n} \rightarrow x$ the strong and the weak convergence to $x$ of a sequence $\left\{x_{n}\right\}$ in a Banach space $X$, respectively. Let $\Gamma_{0}(X)$ denote the class of all lower semi-continuous proper convex functions from $X$ to $(-\infty,+\infty]$. Let $B(x, \delta)$ denote the closed ball of $x \in X$ and radius $\delta>0$. Let $S(X)=\{x \in X:\|x\|=1\}$ be the unit sphere.

A Banach space $X$ is said to be strictly convex if $\frac{1}{2}\|x+y\|<1$ for all $x, y \in S(X)$ and $x \neq y$. The Banach space $X$ is said to be smooth provided

$$
\lim _{t \rightarrow 0} \frac{\|x+t y\|-\|x\|}{t}
$$

exists for each $x, y \in S(X)$. We recall that uniform convexity of $X$ means that for any given $\epsilon>0$, there exists $\delta>0$ such that for all $x, y \in X$ with $\|x\| \leq 1,\|y\| \leq 1$, and $\|x-y\|=\epsilon$, the inequality

$$
\|x+y\| \leq 2(1-\delta)
$$

holds.

A subset $C$ of $X$ is called boundedly compact if for any $\delta>0$ the intersection $C \cap B(0, \delta)$ is empty or compact.

The duality mapping $J: X \rightrightarrows X^{*}$ is defined by

$$
\left.J(x)=\left\{x^{*} \in X^{*}|| x^{*}, x\right\rangle=\left\|x^{*}\right\|^{2}=\|x\|^{2}\right\}, \quad \forall x \in X .
$$

The following basic results concerning the duality mapping are well known $[2,6,7]$ :

(1) $X$ is reflexive if and only if $J$ is surjective;

(2) $X$ is strictly convex if and only if $J$ is injective;

(3) $X$ is smooth if and only if $J$ is single-valued;

(4) if $X$ is smooth, then $J$ is norm-to-weak star continuous;

(5) $J$ is monotone, i.e., $\langle J x-J y, x-y\rangle \geq 0, \forall x, y \in X$;

(6) if $X$ is strictly convex and smooth, then $\langle J x-J y, x-y\rangle=0 \Rightarrow x=y, \forall x, y \in X$;

(7) if a Banach space $X$ is reflexive strictly convex and smooth, then the duality mapping $J^{*}$ from $X^{*}$ into $X$ is the inverse of $J$, that is, $J^{-1}=J^{*}$.

Consider the following envelope function:

$$
e_{V}^{f}\left(x^{*}\right)=\inf _{x \in X}\left\{f(x)+\frac{1}{2} V\left(x^{*}, x\right)\right\}
$$

where $V\left(x^{*}, x\right)=\left\|x^{*}\right\|^{2}-2\left\langle x^{*}, x\right\rangle+\|x\|^{2}$. Since the function $\left(x, x^{*}\right) \rightarrow f(x)+\frac{1}{2} V\left(x^{*}, x\right)$ is lower semicontinuous convex, one sees that $e_{V}^{f}\left(x^{*}\right)$ is lower semicontinuous and convex by Proposition 4.4 in [8].

For every $x^{*} \in X^{*}$, the infimum in (2.1) is achieved at a unique point $\pi_{f}\left(x^{*}\right)$, i.e.,

$$
\pi_{f}\left(x^{*}\right):=\underset{x \in K}{\arg \min }\left\{f(x)+\frac{1}{2} V\left(x^{*}, x\right)\right\} .
$$


The operator $\pi_{f}$ is called the generalized proximity operator. It can be characterized by the inclusion

$$
x^{*}-J \pi_{f}\left(x^{*}\right) \in \partial f\left(\pi_{f}\left(x^{*}\right)\right)
$$

equivalently,

$$
\pi_{f}=(J+\partial f)^{-1}
$$

From (2.3), we easily know that $\pi_{f}$ is maximal monotone by Theorem 2.6.2 in [6]. Observe that when $\rho=1$,

$$
\pi_{K}^{f}\left(x^{*}\right)=\pi_{f+I_{K}}\left(x^{*}\right) .
$$

If, in addition, $\operatorname{dom} f=K$, then $\pi_{K}^{f}\left(x^{*}\right)=\pi_{f}\left(x^{*}\right)$.

Lemma 2.1 ([9]) Let $X$ be a smooth, strictly convex and reflexive Banach space, let $\left\{x_{n}\right\}$ be a sequence in $X$, and $x \in X$. If $\left\langle x_{n}-x, J x_{n}-J x\right\rangle \rightarrow 0$, then $x_{n} \rightarrow x, J x_{n} \rightarrow J x$ and $\left\|x_{n}\right\| \rightarrow\|x\|$.

Lemma 2.2 ([10]) Let $r>0$ be a fixed real number. Then a Banach space $X$ is uniformly convex if and only if there is a continuous, strictly increasing and convex function $g: R^{+} \rightarrow$ $R^{+}$with $g(0)=0$ such that

$$
\|\lambda x+(1-\lambda) y\|^{2} \leq \lambda\|x\|^{2}+(1-\lambda)\|y\|^{2}-\lambda(1-\lambda) g(\|x-y\|), \quad \forall x, y \in B_{r}, 0 \leq \lambda \leq 1,
$$

where $B_{r}=\{x \in X:\|x\| \leq r\}$.

\section{Main results}

Proposition 3.1 Let $f \in \Gamma_{0}(X)$. Then the following hold:

(i) $\left\langle\pi_{f}\left(x^{*}\right)-\pi_{f}\left(y^{*}\right),\left(x^{*}-J \pi_{f}\left(x^{*}\right)\right)-\left(y^{*}-J \pi_{f}\left(y^{*}\right)\right)\right\rangle \geq 0, \forall x^{*}, y^{*} \in X^{*}$;

(ii) $\pi_{f}$ is bounded on each nonempty bounded subset of $C \subset X^{*}$;

(iii) if $\left\{x_{n}^{*}\right\}$ is a sequence in $X^{*}$ such that $x_{n}^{*} \rightarrow x^{*}$, then $\pi_{f}\left(x_{n}^{*}\right) \rightarrow \pi_{f}\left(x^{*}\right)$, $J \pi_{f}\left(x_{n}^{*}\right) \rightarrow J \pi_{f}\left(x^{*}\right)$ and $\left\|\pi_{f}\left(x_{n}^{*}\right)\right\| \rightarrow\left\|\pi_{f}\left(x^{*}\right)\right\| ;$

(iv) if $\operatorname{dom} f$ is a nonempty boundedly compact convex subset, then $\pi_{f}$ is weak-to-norm continuous, that is, if $x_{n}^{*} \rightarrow x^{*}$, then $\pi_{f}\left(x_{n}^{*}\right) \rightarrow \pi_{f}\left(x^{*}\right)$.

Proof (i) Take $x^{*}, y^{*} \in X^{*}$. Then (2.2) yields

$$
\left\langle x^{*}-J \pi_{f}\left(x^{*}\right), \pi_{f}\left(y^{*}\right)-\pi_{f}\left(x^{*}\right)\right\rangle \leq f\left(\pi_{f}\left(y^{*}\right)\right)-f\left(\pi_{f}\left(x^{*}\right)\right)
$$

and

$$
\left\langle y^{*}-J \pi_{f}\left(y^{*}\right), \pi_{f}\left(x^{*}\right)-\pi_{f}\left(y^{*}\right)\right\rangle \leq f\left(\pi_{f}\left(x^{*}\right)\right)-f\left(\pi_{f}\left(y^{*}\right)\right) .
$$

Adding these two inequalities, we obtain

$$
\left\langle\pi_{f}\left(x^{*}\right)-\pi_{f}\left(y^{*}\right),\left(x^{*}-J \pi_{f}\left(x^{*}\right)\right)-\left(y^{*}-J \pi_{f}\left(y^{*}\right)\right)\right\rangle \geq 0 .
$$


(ii) Suppose that $\pi_{f}$ is not bounded on some nonempty bounded subset of $C$. Then there exists a bounded sequence $\left\{x_{n}^{*}\right\} \subset C$ such that $\left\|\pi_{f}\left(x_{n}^{*}\right)\right\| \rightarrow \infty$. Fix $x^{*} \in X^{*}$. From (i), we obtain the following:

$$
\begin{aligned}
\left\|\pi_{f}\left(x_{n}^{*}\right)-\pi_{f}\left(x^{*}\right)\right\|\left\|x_{n}^{*}-x^{*}\right\| & \geq\left\langle\pi_{f}\left(x_{n}^{*}\right)-\pi_{f}\left(x^{*}\right), x_{n}^{*}-x^{*}\right\rangle \\
& \geq\left\langle\pi_{f}\left(x_{n}^{*}\right)-\pi_{f}\left(x^{*}\right), J \pi_{f}\left(x_{n}^{*}\right)-J \pi_{f}\left(x^{*}\right)\right\rangle \\
& =\frac{1}{2}\left(V\left(J \pi_{f}\left(x_{n}^{*}\right), \pi_{f}\left(x^{*}\right)\right)+V\left(J \pi_{f}\left(x^{*}\right), \pi_{f}\left(x_{n}^{*}\right)\right)\right) \\
& \geq\left(\left\|\pi_{f}\left(x_{n}^{*}\right)\right\|-\left\|\pi_{f}\left(x^{*}\right)\right\|\right)^{2} .
\end{aligned}
$$

So, we have $\left\|x_{n}^{*}\right\| \rightarrow \infty$. This is a contradiction.

(iii) Let $\left\{x_{n}^{*}\right\}$ be a sequence in $X^{*}$ such that $x_{n}^{*} \rightarrow x^{*}$. It follows from (ii) that $\left\{\pi_{f}\left(x_{n}^{*}\right)\right\}$ is bounded. From (i), we have

$$
0 \leq\left\langle\pi_{f}\left(x_{n}^{*}\right)-\pi_{f}\left(x^{*}\right), J \pi_{f}\left(x_{n}^{*}\right)-J \pi_{f}\left(x^{*}\right)\right\rangle \leq\left\|\pi_{f}\left(x_{n}^{*}\right)-\pi_{f}\left(x^{*}\right)\right\|\left\|x_{n}^{*}-x^{*}\right\| \rightarrow 0 .
$$

Thus, Lemma 2.1 implies that $\pi_{f}\left(x_{n}^{*}\right) \rightarrow \pi_{f}\left(x^{*}\right), J \pi_{f}\left(x_{n}^{*}\right) \rightarrow J \pi_{f}\left(x^{*}\right)$ and $\left\|\pi_{f}\left(x_{n}^{*}\right)\right\| \rightarrow$ $\left\|\pi_{f}\left(x^{*}\right)\right\|$.

(iv) From (2.2), we know that

$$
\left\langle x_{n}^{*}-J \pi_{f}\left(x_{n}^{*}\right), y-\pi_{f}\left(x_{n}^{*}\right)\right\rangle \leq f(y)-f\left(\pi_{f}\left(x_{n}^{*}\right)\right), \quad \forall y \in \operatorname{dom} f
$$

Since $x_{n}^{*} \rightarrow x^{*},\left\{x_{n}^{*}\right\}$ is bounded. It follows from (ii) that $\left\{\pi_{f}\left(x_{n}^{*}\right)\right\}$ is bounded. Since $\operatorname{dom} f$ is boundedly compact, there exists a subsequence $\left\{x_{n_{i}}^{*}\right\}$ of $\left\{x_{n}^{*}\right\}$ such that

$$
\pi_{f}\left(x_{n_{i}}^{*}\right) \rightarrow \bar{x} \in \operatorname{dom} f \quad \text { as } i \rightarrow+\infty \text {. }
$$

Since $J$ is norm-to-weak star continuous and $f$ is lower semicontinuous, we obtain that

$$
\left\langle x^{*}-J \bar{x}, y-\bar{x}\right\rangle \leq f(y)-f(\bar{x}), \quad \forall y \in \operatorname{dom} f .
$$

Then, by (2.2), we have $\bar{x}=\pi_{f}\left(x^{*}\right)$. Similar to the above arguments, we know that $\pi_{f}\left(x^{*}\right)$ is the unique limit point of $\left\{\pi_{f}\left(x_{n}^{*}\right)\right\}$. Hence, $\pi_{f}\left(x_{n}^{*}\right) \rightarrow \pi_{f}\left(x^{*}\right)$.

With the help of the operator $\pi_{f}$, we can show that the envelope function $e_{V}^{f}$ is Gâteaux differentiable.

Proposition 3.2 Let $f \in \Gamma_{0}(X)$. Then $e_{V}^{f}$ is Gâteaux differentiable and $\nabla e_{V}^{f}\left(x^{*}\right)=J^{*} x^{*}-$ $\pi_{f}\left(x^{*}\right)$.

Proof For any $h \in X^{*}$, by definitions of $e_{V}^{f}$ and $\pi_{f}$, we have

$$
\begin{aligned}
& \frac{e_{V}^{f}\left(x^{*}+t h\right)-e_{V}^{f}\left(x^{*}\right)}{t} \\
& \quad=\frac{f\left(\pi_{f}\left(x^{*}+t h\right)\right)+\frac{1}{2} V\left(\pi_{f}\left(x^{*}+t h\right), x^{*}+t h\right)-f\left(\pi_{f}\left(x^{*}\right)\right)-\frac{1}{2} V\left(\pi_{f}\left(x^{*}\right), x^{*}\right)}{t}
\end{aligned}
$$




$$
\begin{aligned}
& \leq \frac{f\left(\pi_{f}\left(x^{*}\right)\right)+\frac{1}{2} V\left(\pi_{f}\left(x^{*}\right), x^{*}+t h\right)-f\left(\pi_{f}\left(x^{*}\right)\right)-\frac{1}{2} V\left(\pi_{f}\left(x^{*}\right), x^{*}\right)}{t} \\
& =\frac{\frac{1}{2}\left\|x^{*}+t h\right\|^{2}-\frac{1}{2}\left\|x^{*}\right\|^{2}-\left\langle\pi_{f}\left(x^{*}\right), t h\right\rangle}{t} .
\end{aligned}
$$

Since $\nabla\left(\|z\|^{2}\right)=2 J^{*}(z)$, for any $z \in X^{*}$, we get that

$$
\begin{aligned}
\limsup _{t \rightarrow 0} \frac{e_{V}^{f}\left(x^{*}+t h\right)-e_{V}^{f}\left(x^{*}\right)}{t} & \leq \lim _{t \rightarrow 0} \frac{\frac{1}{2}\left\|x^{*}+t h\right\|^{2}-\frac{1}{2}\left\|x^{*}\right\|^{2}}{t}-\left\langle\pi_{f}\left(x^{*}\right), h\right\rangle \\
& =\left\langle J^{*} x^{*}-\pi_{f}\left(x^{*}\right), h\right\rangle .
\end{aligned}
$$

On the other hand,

$$
\begin{aligned}
& \frac{e_{V}^{f}\left(x^{*}+t h\right)-e_{V}^{f}\left(x^{*}\right)}{t} \\
& \quad=\frac{f\left(\pi_{f}\left(x^{*}+t h\right)\right)+\frac{1}{2} V\left(\pi_{f}\left(x^{*}+t h\right), x^{*}+t h\right)-f\left(\pi_{f}\left(x^{*}\right)\right)-\frac{1}{2} V\left(\pi_{f}\left(x^{*}\right), x^{*}\right)}{t} \\
& \quad \geq \frac{f\left(\pi_{f}\left(x^{*}+t h\right)\right)+\frac{1}{2} V\left(\pi_{f}\left(x^{*}+t h\right), x^{*}+t h\right)-f\left(\pi_{f}\left(x^{*}+t h\right)\right)-\frac{1}{2} V\left(\pi_{f}\left(x^{*}+t h\right), x^{*}\right)}{t} \\
& \quad=\frac{\frac{1}{2}\left\|x^{*}+t h\right\|^{2}-\frac{1}{2}\left\|x^{*}\right\|^{2}}{t}-\left\langle\pi_{f}\left(x^{*}+t h\right), h\right\rangle .
\end{aligned}
$$

By Proposition 3.1(iii), we have $\pi_{f}\left(x^{*}+t h\right) \rightarrow \pi_{f}\left(x^{*}\right)$ as $t \rightarrow 0$. Hence, we get that

$$
\begin{aligned}
\liminf _{t \rightarrow 0} \frac{e_{V}^{f}\left(x^{*}+t h\right)-e_{V}^{f}\left(x^{*}\right)}{t} & \geq \lim _{t \rightarrow 0} \frac{\frac{1}{2}\left\|x^{*}+t h\right\|^{2}-\frac{1}{2}\left\|x^{*}\right\|^{2}}{t}-\left\langle\pi_{f}\left(x^{*}+t h\right), h\right\rangle \\
& =\left\langle J^{*} x^{*}-\pi_{f}\left(x^{*}\right), h\right\rangle .
\end{aligned}
$$

This implies that

$$
\lim _{t \rightarrow 0} \frac{e_{V}^{f}\left(x^{*}+t h\right)-e_{V}^{f}\left(x^{*}\right)}{t}=\left\langle J^{*} x^{*}-\pi_{f}\left(x^{*}\right), h\right\rangle .
$$

Hence $e_{V}^{f}$ is Gâteaux differentiable and $\nabla e_{V}^{f}\left(x^{*}\right)=J^{*} x^{*}-\pi_{f}\left(x^{*}\right)$.

In the following, we propose a modification of the iterative method given in [5] and prove that the iterative sequence has a subsequence converging to a solution of (1.5) when $X$ is a smooth and uniformly convex Banach space and $f$ is not necessarily positively homogeneous.

By (2.2), we can easily prove the following result.

Proposition 3.3 Let $f \in \Gamma_{0}(X)$. Then the point $\bar{x} \in \operatorname{dom} f$ is a solution of the variational inequality

$$
\langle A x, y-x\rangle+f(y)-f(x) \geq 0, \quad \forall y \in \operatorname{dom} f
$$

if and only if $\bar{x} \in \operatorname{dom} f$ is a solution of the following inclusion:

$$
x=\pi_{f}(J x-A x) .
$$


The following lemma will be used in proving the convergence of the iterative method for variational inequality problem (1.5).

Lemma 3.1 Let $f \in \Gamma_{0}(X)$. If $f(x) \geq 0$ for all $x \in \operatorname{dom} f$ and $f(0)=0$, then

$$
\left\|\pi_{f}\left(x^{*}\right)\right\| \leq\left\|x^{*}\right\|
$$

Proof From (2.2), we know that

$$
\left\langle x^{*}-J \pi_{f}\left(x^{*}\right), y-\pi_{f}\left(x^{*}\right)\right\rangle \leq f(y)-f\left(\pi_{f}\left(x^{*}\right)\right), \quad \forall y \in \operatorname{dom} f .
$$

Noticing that $f(x) \geq 0$ for all $x \in \operatorname{dom} f$ and $f(0)=0$, it follows that

$$
\left\langle x^{*}-J \pi_{f}\left(x^{*}\right),-\pi_{f}\left(x^{*}\right)\right\rangle \leq-f\left(\pi_{f}\left(x^{*}\right)\right) \leq 0 .
$$

Hence,

$$
\left\|\pi_{f}\left(x^{*}\right)\right\|^{2} \leq\left\langle x^{*}, \pi_{f}\left(x^{*}\right)\right\rangle
$$

and hence

$$
\left\|\pi_{f}\left(x^{*}\right)\right\| \leq\left\|x^{*}\right\| .
$$

Proposition 3.4 Let $X$ be a smooth and uniformly convex Banach space. Let $A: X \rightarrow X^{*}$ be a norm-to-weak continuous operator. Suppose that $f \in \Gamma_{0}(X)$ and $\operatorname{dom} f$ is nonempty boundedly compact convex. Suppose that

(i) $f(x) \geq 0$ for all $x \in \operatorname{dom} f$ and $f(0)=0$;

(ii) for any $x \in \operatorname{dom} f$,

$$
\|J x-A x\| \leq\|x\| .
$$

Let $x_{0} \in \operatorname{dom} f$ and the sequence $\left\{x_{n}\right\}$ be generated by the following iteration scheme:

$$
x_{n+1}=\left(1-\alpha_{n}\right) x_{n}+\alpha_{n} \pi_{f}\left(J x_{n}-A x_{n}\right),
$$

where $\left\{\alpha_{n}\right\}$ satisfies the conditions:

(a) $0 \leq \alpha_{n} \leq 1$ for all $n=0,1,2, \ldots$;

(b) $\sum_{n=0}^{+\infty} \alpha_{n}\left(1-\alpha_{n}\right)=+\infty$.

Then generalized variational inequality (1.5) has a solution $\bar{x} \in \operatorname{dom} f$, and there exists a subsequence $\left\{x_{n_{i}}\right\}$ of $\left\{x_{n}\right\}$ such that $x_{n_{i}} \rightarrow \bar{x}$ as $i \rightarrow \infty$.

Proof By (3.3), we have

$$
\left\|\pi_{f}\left(J x_{n}-A x_{n}\right)\right\| \leq\left\|J x_{n}-A x_{n}\right\| .
$$

By (3.4) and condition (ii), we obtain

$$
\left\|x_{n+1}\right\| \leq\left(1-\alpha_{n}\right)\left\|x_{n}\right\|+\alpha_{n}\left\|\pi_{f}\left(J x_{n}-A x_{n}\right)\right\| \leq\left\|x_{n}\right\| .
$$


Then $\left\{x_{n}\right\}$ and $\left\{\pi_{f}\left(J x_{n}-A x_{n}\right)\right\}$ are bounded. Hence, by Lemma 2.2, there exists a continuous, strictly increasing and convex function $g: R^{+} \rightarrow R^{+}$with $g(0)=0$ such that

$$
\begin{aligned}
\left\|x_{n+1}\right\|^{2}= & \left\|\left(1-\alpha_{n}\right) x_{n}+\alpha_{n} \pi_{f}\left(J x_{n}-A x_{n}\right)\right\|^{2} \\
\leq & \left(1-\alpha_{n}\right)\left\|x_{n}\right\|^{2}+\alpha_{n}\left\|\pi_{f}\left(J x_{n}-A x_{n}\right)\right\|^{2} \\
& -\alpha_{n}\left(1-\alpha_{n}\right) g\left(\left\|x_{n}-\pi_{f}\left(J x_{n}-A x_{n}\right)\right\|\right) .
\end{aligned}
$$

It follows from (3.5), (3.4) and condition (ii) that

$$
\left\|x_{n+1}\right\|^{2} \leq\left(1-\alpha_{n}\right)\left\|x_{n}\right\|^{2}+\alpha_{n}\left\|x_{n}\right\|^{2}-\alpha_{n}\left(1-\alpha_{n}\right) g\left(\left\|x_{n}-\pi_{f}\left(J x_{n}-A x_{n}\right)\right\|\right) .
$$

That is,

$$
\left\|x_{n+1}\right\|^{2} \leq\left\|x_{n}\right\|^{2}-\alpha_{n}\left(1-\alpha_{n}\right) g\left(\left\|x_{n}-\pi_{f}\left(J x_{n}-A x_{n}\right)\right\|\right) .
$$

Taking the sum for $n=0,1,2, \ldots, m$ in (3.6), we get

$$
\begin{aligned}
& \sum_{n=0}^{m} \alpha_{n}\left(1-\alpha_{n}\right) g\left(\left\|x_{n}-\pi_{f}\left(J x_{n}-A x_{n}\right)\right\|\right) \\
& \quad \leq\left\|x_{0}\right\|^{2}-\left\|x_{m+1}\right\|^{2} \\
& \quad \leq\left\|x_{0}\right\|^{2} .
\end{aligned}
$$

Hence,

$$
\sum_{n=0}^{+\infty} \alpha_{n}\left(1-\alpha_{n}\right) g\left(\left\|x_{n}-\pi_{f}\left(J x_{n}-A x_{n}\right)\right\|\right)<+\infty
$$

Due to the condition $\sum_{n=0}^{+\infty} \alpha_{n}\left(1-\alpha_{n}\right)=+\infty$, we may assume, without loss of generality, that

$$
g\left(\left\|x_{n}-\pi_{f}\left(J x_{n}-A x_{n}\right)\right\|\right) \rightarrow 0 \quad \text { as } n \rightarrow+\infty .
$$

Applying the properties of $g$, we can deduce that

$$
\left\|x_{n}-\pi_{f}\left(J x_{n}-A x_{n}\right)\right\| \rightarrow 0 \quad \text { as } n \rightarrow+\infty .
$$

Since $\operatorname{dom} f$ is boundedly compact, there exists a subsequence $\left\{x_{n_{i}}\right\}$ of $\left\{x_{n}\right\}$ such that

$$
x_{n_{i}} \rightarrow \bar{x} \in \operatorname{dom} f \quad \text { as } i \rightarrow+\infty .
$$

Since $A$ is norm-to-weak continuous and $J$ is norm-to-weak star continuous, we get that

$$
J x_{n_{i}}-A x_{n_{i}} \rightarrow J \bar{x}-A \bar{x} \quad \text { as } i \rightarrow+\infty .
$$


Since $\pi_{f}$ is weak-to-norm continuous by Proposition 3.1(iv),

$$
\pi_{f}\left(J x_{n_{i}}-A x_{n_{i}}\right) \rightarrow \pi_{f}(J \bar{x}-A \bar{x}) \quad \text { as } i \rightarrow+\infty .
$$

Hence, (3.8) yields

$$
\bar{x}=\pi_{f}(J \bar{x}-A \bar{x}) .
$$

Now it follows from Proposition 3.3 that $\bar{x}$ is a solution of generalized variational inequality (1.5).

\section{Application}

Let $f \in \Gamma_{0}(X)$ and let $g: X \rightarrow \mathbb{R}$ be a convex and Gâteaux differentiable function. Consider the optimization problem

$$
\min _{x \in X} f(x)+g(x)
$$

We denote by $\operatorname{Sol}(\mathrm{P})$ the solution set of problem $(\mathrm{P})$. Despite its simplicity, problem $(\mathrm{P})$ has been shown to cover a wide range of apparently unrelated signal recovery formulations (see $[11,12])$.

Notice that

$$
\begin{aligned}
\bar{x} \in \operatorname{Sol}(\mathrm{P}) & \Leftrightarrow \quad 0 \in \partial f(\bar{x})+\nabla g(\bar{x}) \\
& \Leftrightarrow \quad-\nabla g(\bar{x}) \in \partial f(\bar{x}) \\
& \Leftrightarrow \quad\langle\nabla g(\bar{x}), y-\bar{x}\rangle+f(y)-f(\bar{x}) \geq 0, \quad \forall y \in X .
\end{aligned}
$$

Note that if $g$ is convex and Gâteaux differentiable, then $\nabla g$ is norm-to-weak continuous from $X$ to $X^{*}$ by Corollary 3.1 in [13]. Therefore, as an application of Proposition 3.4, we have the following result.

Proposition 4.1 Let $X$ be a smooth and uniformly convex Banach space. Let $g: X \rightarrow \mathbb{R}$ be convex and Gâteaux differentiable. Suppose that $f \in \Gamma_{0}(X)$ and $\operatorname{dom} f$ is a nonempty boundedly compact convex subset of $X$. Suppose that

(i) $f(x) \geq 0$ for all $x \in \operatorname{dom} f$ and $f(0)=0$;

(ii) for any $x \in \operatorname{dom} f$,

$$
\|J x-\nabla g(x)\| \leq\|x\| .
$$

Let $x_{0} \in \operatorname{dom} f$ and the sequence $\left\{x_{n}\right\}$ be generated by the following iteration scheme:

$$
x_{n+1}=\left(1-\alpha_{n}\right) x_{n}+\alpha_{n} \pi_{f}\left(J x_{n}-\nabla g\left(x_{n}\right)\right) \text {, }
$$

where $\left\{\alpha_{n}\right\}$ satisfies the conditions:

(a) $0 \leq \alpha_{n} \leq 1$ for all $n=0,1,2, \ldots$;

(b) $\sum_{n=0}^{+\infty} \alpha_{n}\left(1-\alpha_{n}\right)=+\infty$.

Then problem $(\mathrm{P})$ has a solution $\bar{x}$ and there exists a subsequence $\left\{x_{n_{i}}\right\}$ of $\left\{x_{n}\right\}$ such that $x_{n_{i}} \rightarrow \bar{x}$ as $i \rightarrow \infty$. 


\section{Concluding remark}

This paper has improved the iterative method of Wu and Huang [5] for solving generalized variational inequality problem (1.5), several results regarding the generalized proximity operator and its relations with the envelope function are presented. In addition, it is shown that under an appropriate assumption some optimization problem can be transformed into (1.5) and then the iterative method can be applied.

\section{Competing interests}

The author declares that they have no competing interests.

\section{Acknowledgements}

The work was supported by the Scientific Technology Program of the Educational Department Heilongjiang Province (No. 12511161) and the National Natural Sciences Grant (No. 11071052).

Received: 30 August 2013 Accepted: 11 November 2013 Published: 05 Dec 2013

\section{References}

1. Alber, Y: Generalized projection operators in Banach spaces: properties and applications. In: Proceedings of the Israel Seminar Ariel, Israel, Funct. Differ. Equ., vol. 1, pp. 1-21 (1994)

2. Alber, Y: Metric and generalized projection operators in Banach spaces: properties and applications. In: Kartsatos, A (ed.) Theory and Applications of Nonlinear Operators of Accretive and Monotone Type, pp. 15-50. Dekker, New York (1996)

3. Li, J: The generalized projection operator on reflexive Banach spaces and its applications. J. Math. Anal. Appl. 306, 55-71 (2005)

4. Zeng, LC, Yao, JC: Existence theorems for variational inequalities in Banach spaces. J. Optim. Theory Appl. 36, 483-497 (2006)

5. Wu, K-Q, Huang, N-J: Properties of the generalized $f$-projection operator and its applications in Banach spaces. Comput. Math. Appl. 54, 399-406 (2007)

6. Barbu, V, Precupanu, T: Convexity and Optimization in Banach Spaces. Springer, New York (2012)

7. Takahashi, W: Convex Analysis and Approximation of Fixed Points. Mathematical Analysis Series, vol. 2. Yokohama Publishers, Yokohama (2000)

8. Bonnans, JF, Shapiro, A: Perturbation Analysis of Optimization Problems. Springer, New York (2000)

9. Aoyama, K, Kohsaka, F, Takahashi, W: Three generalizations of firmly nonexpansive mappings: their relations and continuity properties. J. Nonlinear Convex Anal. 10, 131-147 (2009)

10. Xu, H-K: Inequalities in Banach spaces with applications. Nonlinear Anal. 16, 1127-1138 (1991)

11. Combettes, PL: Inconsistent signal feasibility problems: least-squares solutions in a product space. IEEE Trans. Signal Process. 42, 2955-2966 (1994)

12. Combettes, PL, Wajs, VR: Signal recovery by proximal forward-backward splitting. Multiscale Model. Simul. 4, 1168-1200 (2005)

13. Asplund, E, Rockafellar, RT: Gradient of convex functions. Trans. Am. Math. Soc. 139, 443-467 (1969)

10.1186/1029-242X-2013-574

Cite this article as: Guan: An iterative method for variational inequality problems. Journal of Inequalities and Applications 2013, 2013:574 